\title{
SISTEMAS DE ROTAÇÃO DE CULTURAS DE MILHO, ALGODÃO E SOJA E SEU EFEITO SOBRE A PRODUTIVIDADE ${ }^{1}$ )
}

\author{
HIPÓlITO ASSUNÇÃO ANTONIO MASCARENHAS $\left({ }^{2,6}\right)$, VIOLETA NAGAI $\left({ }^{3}\right)$,

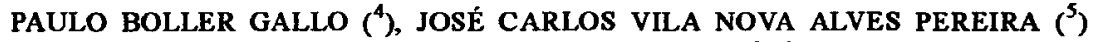 \\ e ROBERTO TETSUO TANAKA $\left({ }^{2,6}\right)$
}

\begin{abstract}
RESUMO
Nos períodos de 1981/82 a 1988/89 em Ribeirão Preto e de 1981/82 a 1990/91 em Mococa, estudaram-se sistemas de rotação de culturas de soja, milho e algodão segundo os esquemas: I: rotação algodão, milho e soja; II: dois anos de algodão e um de soja; III: dois anos de milho e um de soja; IV: dois anos de soja e um de milho e V: dois anos de soja e um de algodão, além das monoculturas de soja, de milho e algodão. Os resultados mostraram aumento da produtividade de grãos de milho com a adoção dos sistemas de rotação quando comparados ao monocultivo, não ocorrendo o mesmo nas culturas de algodão e soja.
\end{abstract}

Termos de inderação: algodão, milho, soja, sistemas de rotação.

\section{ABSTRACT \\ ROTATION SYSTEMS OF CORN, COTTON AND SOYBEAN AND ITS EFFECT ON YIELD}

\begin{abstract}
During the period of $1981 / 82$ to $1988 / 89$ at Ribeirão Preto and 1981/82 to $1990 / 91$ at Mococa, São Paulo State, Brazil, rotation systems were studied, with corn, cotton and soybeans using the following scheme: I - annual rotation of cotton, corn and soybeans; II - two years of cotton and one year of soybeans; III - two years of corn and one year of soybeans; IV - two years of soybeans and one year of corn; V - two years of soybeans and one year of cotton; and the monoculture of corn, cotton and soybeans. The results showed that at the two localities there was an increase in the yield of corn whatever the scheme of rotation utilized. On the other hand, for both cotton and soybeans no significant increase in the yield was observed with rotations.
\end{abstract}

Index terms: corn, cotton, soybeans, systems of the rotation.

(1) Trabalho apresentado no XXIV Congresso Brasileiro de Ciência do Solo, 25 a 31 de julho de 1993, em Goiânia (GO). Recebido para publicação em 6 de outubro e aceito em 11 de maio de 1993. Trabalho parcialmente financiado pela EMBRAPA e pela COMOVE.

$\left({ }^{2}\right)$ Seção de Leguminosas, Instituto Agronômico (IAC), Caixa Postal 28, 13001-970, Campinas (SP).

(3) Seção de Técnica Experimental e Cálculo, IAC.

(4) Estação Experimental de Mococa, IAC.

(5) Estação Experimental de Ribeirão Preto, IAC.

( ${ }^{6}$ Com Bolsa de Pesquisa do CNPq. 


\section{INTRODUÇÃO}

As culturas de soja, milho e algodão são economicamente importantes nas regiōes da média e da alta Mojiana (SP), havendo expansão dos seus cultivos na década de 70 . Entretanto, na década de 80, devido a incentivo para produção de açúcar $\mathrm{e}$ álcool, houve substancial expansão da área com cana-de-açúcar, ocupando solos férteis antes destinados àquelas culturas. Nessa última década, em termos globais, não houve grande aumento de área, apenas expansões nas de milho, sempre em prejuízo das outras duas culturas (algodão e soja) e sem avançar na área canavieira.

O benefício gerado pelas leguminosas a outras culturas que as sucedem, ou com elas são cultivadas em consorciação, foi constatado nos EUA por Stickler et al. (1959) e Sutherland et al. (1961). Em estudos realizados em Ribeirão Preto e Pindorama em 1947/52, Neme (1960) mostrou o efeito vantajoso sobre a produtividade do milho na rotação com Crotalaria juncea, guandu (Cajanus cajan (L.) Mill sp.) e mucuna-preta (Stilozobium aterrimum Piper \& Tracy). Em latossolo roxo (cerrado recém-recuperado), Mascarenhas et al. (1978) observaram que o milho cultivado em sucessão à soja por um a quatro anos consecutivos apresentou pequeno aumento de produção pela aplicação de nitrogênio mineral em cobertura, inferindo que os restos de culturas, raízes e nódulos incorporados ao solo, após a colheita da soja, constituem fontes suficientes de nitrogênio para essa gramínea. Observaram, ainda, aumento de produção de grão diretamente proporcional ao tempo de cultivo anterior com soja. Esses dados foram confirmados por Gallo et al. (1981). Trabalhos realizados por Oliveira et al. (1979) com trigo e Pereira et al. $(1979,1988)$ com arroz e algodão, sendo as três culturas cultivadas após soja, demonstraram que, quando bem nodulada, a soja constituiu fonte suficiente de nitrogênio para tais culturas.

Bertoni et al. (1972) relataram experimentos de rotação trienal de milho, algodão e soja, desenvolvidos em Pindorama e Ribeirão Preto em 1944/58: o milho em rotação produziu $28 \%$ em Pindorama e $61 \%$ em Ribeirão Preto a mais do que em monocultivo; os acréscimos foram maiores para a soja em rotação em cerca de $47 \%$ (Pindorama) e $76 \%$
(Ribeirão Preto). Por outro lado, o algodão em rotação teve queda no seu rendimento quando comparado ao monocultivo.

O principal objetivo deste trabalho foi verificar a produtividade das culturas de algodão, milho e soja em sistema de rotação.

\section{MATERIAL E MÉTODOS}

Os experimentos foram desenvolvidos em 1981/89 em Ribeirão Preto, num latossolo roxo distrófico, e em 1981/91 em Mococa, num podzólico vermelho-amarelo orto. Os resultados das análises químicas do solo por ocasião da escolha das áreas para o experimento são apresentados no quadro 1. Nas duas localidades, aplicou-se calcário dolomitico na base de 6 t/ha para reduzir a acidez do solo e propiciar condições ao melhor desenvolvimento de três culturas: soja, milho e algodão.

No quadro 2, encontram-se as seqüências dos três cultivos da rotação, as quais foram repetidas no quarto e no sétimo ano agrícola. Para milho e algodão, a parcela consistiu em seis linhas de $8 \mathrm{~m}$ com espaçamento de $1 \mathrm{~m}$ entre as linhas. Para a soja, a parcela consistiu em dez linhas de $8 \mathrm{~m}$ com espaçamento de $0,60 \mathrm{~m}$ entre as linhas.

Os 18 tratamentos foram estudados em delineamento de blocos ao acaso com quatro repetições. No algodão e no milho, colheram-se as duas linhas centrais de $6 \mathrm{~m}$, excluindo $1 \mathrm{~m}$ de cada extremidade. Para soja, as quatro linhas de $5 \mathrm{~m}$, excluindo 1,50 m de cada extremidade. Após a colheita da área útil do algodão, todas as plantas foram retiradas da parcela. Nas outras duas culturas, as plantas da área útil foram retiradas da parcela e as da bordadura, incorporadas uniformemente ao solo com aração e gradagem. As parcelas do algodão também foram submetidas ao mesmo manejo do solo descrito. $O$ cultivar de soja utilizado foi o IAC-9 (semitardio) e tolerante a Meloidogyne incognita; o cultivar de milho foi o Hmd-7974 e o de algodão, o IAC-19, também tolerante a $M$. incognita.

As adubações anuais para soja, milho e algodão foram, respectivamente, de $300 \mathrm{~kg} / \mathrm{ha}$ da fórmula $0-20-20,400 \mathrm{~kg} / \mathrm{ha}$ da fórmula 4-15-15 e $350 \mathrm{~kg} / \mathrm{ha}$ da fórmula 4-20-20. As doses dos nutrientes aplicadas 
foram determinadas, levando-se em consideração que não fossem limitantes nem tóxicas para o desenvolvimento normal das plantas. Para suprir as necessidades em nitrogênio, as sementes de soja foram inoculadas antes da semeadura, enquanto, para milho e algodão, aplicaram-se, respectivamente, 60 e 40 $\mathrm{kg} / \mathrm{ha}$ de $\mathrm{N}$ na forma de sulfato de amônio, 35 dias após a germinação.
Foram feitas análises da variância por ano e conjunta, tendo sido empregado o teste $F$ para estudo dos contrastes de interesse. Aplicou-se, também, na análise dos dados de produção de milho, o teste de combinação de probabilidades proposto por Fisher e citado por Conagin et al. (1993), utilizando o teste de Bonferroni para estudo dos contrastes com cultivo contínuo.

Quadro 1.Análise química do solo antes da aplicação de calcário e da implantação dos experimentos

\begin{tabular}{|c|c|c|c|c|c|c|c|}
\hline Localidade & $\mathrm{pH}\left({ }^{1}\right)$ & M.O. & $\mathbf{P}^{(2)}$ & $\mathbf{K}$ & $\mathrm{Ca}$ & $\mathbf{M g}$ & Al \\
\hline & & $\%$ & $\mu \mathrm{g} / \mathrm{cm}^{3}$ & $\ldots$ & $-\mathbf{m} \epsilon$ & $\mathrm{m}^{3}$ & \\
\hline Mococa & 4,4 & 3,5 & 2,3 & 0,10 & 1,0 & 0,5 & 0,2 \\
\hline Ribeirão Preto & 4,8 & 3,7 & 3,7 & 0,13 & 1,0 & 0,3 & 0,5 \\
\hline
\end{tabular}

() Medido em água ( $\left.{ }^{2}\right)$ Extrator de ácído sulfúrico.

Quadro 2. Esquema dos tratamentos utilizados na avaliação dos sistemas de rotação de culturas

\begin{tabular}{lllll}
\multirow{2}{*}{ Sistema } & Tratamento & \multicolumn{3}{c}{ Ano agricola } \\
\cline { 3 - 5 } & & $1981 / 82$ & $1982 / 83$ & $1983 / 84$ \\
\hline I & 1 & Algodão & Milho & Soja \\
& 2 & Milho & Soja & Algodão \\
II & 3 & Soja & Algodão & Milho \\
& 4 & Algodão & Algodão & Soja \\
& 5 & Algodão & Soja & Algodão \\
III & 6 & Soja & Algodão & Algodão \\
& 7 & Milho & Milho & Soja \\
& 8 & Milho & Soja & Milho \\
IV & 9 & Soja & Milho & Milho \\
& 10 & Soja & Soja & Milho \\
& 11 & Soja & Milho & Soja \\
V & 12 & Milho & Soja & Soja \\
& 13 & Soja & Soja & Algodão \\
& 14 & Soja & Algodão & Soja \\
Monocultivo & 15 & Algodão & Soja & Soja \\
Monocultivo & 16 & Soja & Soja & Soja \\
Monocultivo & 17 & Milho & Milho & Milho \\
\hline
\end{tabular}




\section{RESULTADOS E DISCUSSÃo}

\subsection{Estação Experimental de Mococa}

A seca prolongada do ano agrícola 1982/83 provocou a perda do experimento e, conseqüentemente os dados do primeiro ciclo (1981/84) foram excluídos. Em 1984/85 foi usado girassol em substituição ao algodão em vista da proibição do seu cultivo na região da média Mojiana por causa da praga do bicudo.

\section{Milho}

$\mathrm{O}$ quadro 3 mostra as produtividades anuais de milho, a indicação de significância do teste F na análise estatística e o valor de $\chi^{2}$ no teste de combinação de probabilidades. Nota-se que o melhor rendimento relativo foi para 0 tratamento soja-soja-milho, com um aumento na produtividade de $19 \%$ quando comparado com os monocultivos. Dados semelhantes foram observados em Pindorama por Bertoni et al. (1972). Qualquer que tenha sido o sistema de rotação, neste o milho produziu mais do que em monocultivo, como mostram os resultados de aplicação do teste de combinação de probabilidades. $O$ sistema soja-soja-milho e soja-milho-milho apresentaram maiores produçð̃es do que soja-algodão-milho e milho-soja-milho, em três dos oito anos agrícolas. Os restos de cultura de soja e seu sistema radicular acrescidos da nodulação, conforme apontado por Pereira et al. (1988), devem ter fornecido o nitrogênio necessário para o aumento de produção.

Quadro 3. Produtividade de milho em rotação com algodão e soja, resultados do teste $F$ na análise da variância e do teste de combinação de probabilidades $\left(\chi^{2}\right)$, em Mococa (SP)

\begin{tabular}{|c|c|c|c|c|c|c|c|c|c|}
\hline \multirow{2}{*}{ Rotação } & \multicolumn{7}{|c|}{ Anos agrícolas } & \multirow{2}{*}{ Média } & \multirow{2}{*}{$\begin{array}{r}\text { Produção } \\
\text { relativa }\end{array}$} \\
\hline & $84 / 85$ & $85 / 86$ & $86 / 87$ & $87 / 88$ & $88 / 89$ & $89 / 90$ & $90 / 91$ & & \\
\hline & \multicolumn{8}{|c|}{$-\mathrm{kg} / \mathrm{ha}$} & $\%$ \\
\hline S-A-M & 5.561 & 6.264 & 5.715 & 4.307 & 5.693 & 2.811 & 5.233 & 5.083 & 109 \\
\hline S-M-M & 5.790 & 5.402 & 5.183 & 4.515 & 6.050 & 2.714 & 5.886 & 5.077 & 109 \\
\hline M-S-M & 5.768 & 5.364 & 5.740 & 4.581 & 5.038 & 3.745 & 5.073 & 5.044 & 108 \\
\hline S-S-M & 6.349 & 6.142 & 6.040 & 4.820 & 5.965 & 3.998 & 5.677 & 5.570 & 119 \\
\hline Milho-C & 6.080 & 5.453 & 5.277 & 3.875 & 4.691 & 2.782 & 4.480 & 4.663 & 100 \\
\hline Média & 5.909 & 5.725 & 5.591 & 4.420 & 5.507 & 3.210 & 5.270 & 5.087 & \\
\hline CV\% & 12,4 & 6,5 & 12,6 & 12,3 & 7,3 & 9,5 & 7,4 & 8,8 & \\
\hline
\end{tabular}

\section{Contrastes}

C vs. rotação

S-A-M vs. S-S-M

S-M-M vs. M-S-M

Anos $\mathrm{x}$ rotação

S-A-M vs. C

S-M-M vs. C

M-S-M vs. C

S-S-M vs. C
Análise conjunta

\begin{tabular}{|c|c|c|c|c|c|c|c|}
\hline ns & ns & $*$ & $*$ & $*$ & * & $*$ & * \\
\hline $\mathbf{n s}$ & ns & * & ns & ns & * & $\mathrm{ns}$ & * \\
\hline ns & ns & * & ns & $*$ & $*$ & ns & ns \\
\hline \multirow[t]{5}{*}{-} & - & - & - & - & - & - & * \\
\hline & & & & & & & $38,89^{*}$ \\
\hline & & & & & & & $31,01^{*}$ \\
\hline & & & & & & & $35,03^{*}$ \\
\hline & & & & & & & $79,37^{*}$ \\
\hline
\end{tabular}

$\mathbf{M}=$ milho; $\mathbf{S}=$ soja $; \mathbf{A}=$ algodão $\mathbf{C}=$ plantio contínuo. 


\section{Algodão}

Os dados apresentados no quadro 4 evidenciaram que não houve diferenças significativas entre os sistemas de rotação e monocultivo, quando avaliados mediante produtividade do algodão. Pela produção relativa, nota-se um aumento de $4 \%$ no sistema de produção algodão-algodão-soja (A-A-S) e uma redução de $6 \%$ nos sistemas soja-algodão-soja (S-A-S) e algodão-soja-soja (A-S-S) quando comparados com algodão em plantio contínuo. Dádos semelhantes foram obtidos por Bertoni et al. (1972) tanto em Pindorama como em Ribeirão Preto.

\section{Soja}

No quadro 5 , observam-se as produtividades de soja em rotação e em monocultivo. Nota-se, pelas produçð̃es relativas, que soja no sistema milho-soja-soja produziu $5 \%$ a mais do que soja em plantio contínuo, enquanto no sistema milho-milho-soja, houve um acréscimo de apenas $2 \%$. De modo geral, houve pequeno acréscimo pela rotação. Os contrastes permitem visualizar que nenhum sistema de rotação revelou diferenças em relação ao monocultivo. Tanto em Ribeirão Preto como em Pindorama, Bertoni et al. (1972) obtiveram aumentos relativos de soja em rotação de 47 e $76 \%$ respectivamente em relação ao contínuo. Esses aumentos talvez tenham sido devidos ao uso de diferentes variedades de soja (Abura, Otootan, Morro Agudo e Araçatuba) na experimentação.

\subsection{Estação Experimental de Ribeiråo Preto}

Os dados do primeiro ciclo (1981/84) das rotações foram excluídos devido ao excesso de chuva que prejudicou o desenvolvimento das três culturas.

\section{Milho}

Como nos dados de Mococa, as produtividades obtidas (Quadro 6) em todos os anos (exceto em 1988/ 189) com o monocultivo de milho foram sempre menores do que com o milho em rotação. Pelo teste de

Quadro 4. Produtividade de algodão em caroço, em rotação com milho e soja, resultados do teste F na análise da variância e do teste de combinação de probabilidades $\left(\chi^{2}\right)$ em Mococa (SP)

\begin{tabular}{|c|c|c|c|c|c|c|c|c|}
\hline \multirow{2}{*}{ Rotação } & \multicolumn{6}{|c|}{ Anos agrícolas } & \multirow{2}{*}{ Média } & \multirow{2}{*}{$\begin{array}{r}\text { Produção } \\
\text { relativa }\end{array}$} \\
\hline & $85 / 86$ & $86 / 87$ & $87 / 88$ & $88 / 89$ & $89 / 90$ & $90 / 91$ & & \\
\hline & \multicolumn{6}{|c|}{ - kg/ha } & $\overline{-}$ & $\%$ \\
\hline M-S-A & 2.564 & 2.430 & 1.695 & 3.168 & 1.411 & 1.396 & 2.110 & 98 \\
\hline S-A-A & 2.727 & 2.994 & 1.595 & 3.496 & 1.455 & 1.097 & 2.227 & 104 \\
\hline A-S-A & 2.808 & 2.629 & 1.474 & 3.021 & 1.314 & 842 & 2.015 & 94 \\
\hline S-S-A & 2.448 & 2.618 & 1.610 & 2.869 & 1.376 & 1.247 & 2.028 & 94 \\
\hline Algodão-C & 2.936 & 2.426 & 1.927 & 3.368 & 1.496 & 950 & 2.151 & 100 \\
\hline Média & 2.697 & 2.619 & 1.660 & 3.179 & 1.416 & 1.106 & 2.106 & \\
\hline CV\% & 13,1 & 12,8 & 16,7 & 22,3 & 14,2 & 22,9 & 18,6 & \\
\hline \multicolumn{5}{|l|}{ Contrastes } & \multicolumn{3}{|c|}{ Análise conjunta } & \\
\hline C vs. rotação & ns & ns & ns & ns & ns & ns & ns & \\
\hline M-S-A. vs. S-S-A & ns & ns & ns & ns & ns & ns & ns & \\
\hline S-A-A. vs. A-S-A & ns & ns & ns & ns & ns & ns & ns & \\
\hline Anos X rotação & - & - & - & - & - & - & - & \\
\hline
\end{tabular}

$\mathrm{M}=$ milho; $\mathbf{S}=$ soja; $\mathrm{A}=$ algodåo; $\mathbf{C}=$ plantio contínuo. 
combinação de probabilidades, observa-se que, no conjunto dos anos, os diferentes tipos de rotação foram estatisticamente superiores ao monocultivo. Examinando a produção relativa no conjunto de anos em comparação com milho contínuo, este em rotação soja-algodão-milho produziu $23 \%$ a mais e, nas três seguintes rotações, produziu em média $13 \%$ a mais que no monocultivo, comprovando os benefícios que os sistemas de rotação ou de sucessão podem trazer à cultura subseqüente ao milho.

Nessa mesma localidade, Bertoni et al. (1972) observaram $61 \%$ de aumento pela rotação em comparação ao monocultivo.

\section{Algodão}

Como em Mococa (Quadro 4), também em Ribeirão Preto (Quadro 7), os sistemas de rotação de cultura mostraram-se ineficientes para aumentar a produtividade do algodão, quando comparado ao seu monocultivo.

A ausência de resposta do algodoeiro à rotação de culturas provavelmente se deva mais a sua pequena exigência ao nitrogênio, ao contrário do milho. Isso significa que a adubação daquele nutriente na dose aplicada de $40 \mathrm{~kg} / \mathrm{ha}$ já poderia ser suficiente para alcançar a produtividade apresentada no quadro 4 .

Pereira et al. (1988) também verificaram que o algodoeiro não respondia ao efeito residual da soja, tanto quando adubado como quando não adubado com nitrogênio, e também não diferiu da testemunha o monocultivo com aquele elemento.

\section{Soja}

Avaliando-se os dados do quadro 8, nota-se que não foram significativos os contrastes estudados.

Quadro 5. Produtividade de soja em rotação com algodão e milho, resultados do teste $F$ na análise da variância e do teste de combinação de probabilidades $\left(\chi^{2}\right)$, em Mococa (SP)

\begin{tabular}{|c|c|c|c|c|c|c|c|c|c|}
\hline \multirow{2}{*}{ Rotação } & \multicolumn{7}{|c|}{ Anos agrícolas } & \multirow{2}{*}{ Média } & \multirow{2}{*}{$\begin{array}{c}\text { Produção } \\
\text { relativa }\end{array}$} \\
\hline & $84 / 85$ & $85 / 86$ & $86 / 87$ & $87 / 88$ & $88 / 89$ & $89 / 90$ & $90 / 91$ & & \\
\hline & \multicolumn{7}{|c|}{ kg/ha } & & $\%$ \\
\hline A-M-S & 2.868 & 2.528 & 2.455 & 2.334 & 2.541 & 1.732 & 1.600 & 2.294 & 100 \\
\hline$A-A-S$ & 2.912 & 27.2 & 2.512 & 2.020 & 2.990 & 1.508 & 1.556 & 2.316 & 101 \\
\hline M-M-S & 2.459 & 2041 & 2.668 & 2.226 & 2.817 & 1.925 & 1.618 & 2.336 & 102 \\
\hline M-S-S & 2.977 & 2.598 & 2.391 & 2.512 & 2.901 & 1.581 & 1.886 & 2.407 & 105 \\
\hline S-M-S & 2.764 & 2.293 & 2.296 & 2.326 & 2.660 & 1.570 & 1.662 & 2.224 & 97 \\
\hline A-S-S & 2.658 & 2.519 & 2.321 & 2.465 & 2.940 & 1.523 & 1.727 & 2.308 & 101 \\
\hline S-A-S & 2.976 & 2.380 & 2.766 & 2.315 & 2.450 & 1.454 & 1.427 & 2.253 & 98 \\
\hline Soja-C & 2.984 & 2.515 & 2.674 & 2.237 & 2.411 & 1.620 & 1.614 & 2.294 & 100 \\
\hline Média & 2.825 & 2.523 & 2.510 & 2.329 & 2.714 & 1.614 & 1.636 & 2.304 & \\
\hline $\mathrm{CV} \%$ & 10,3 & 13,8 & 17,1 & 11,2 & 12,3 & 18,3 & 13,0 & 13,7 & \\
\hline \multicolumn{5}{|l|}{ Contrastes } & \multicolumn{3}{|c|}{ Análise conjunta } & & \\
\hline C vs. rotação & ns & ns & ns & ns & ns & ns & & & \\
\hline$A-M-S$ vs. A-A-S + M-M-S & ns & ns & ns & ns & ns & ns & & & \\
\hline M-S-S vs. S-M-S & ns & ns & ns & ns & ns & ns & & & \\
\hline A-S-S vs. $M-M-S$ & ns & ns & ns & ns & ns & ns & & & \\
\hline Anos $\mathrm{X}$ rotação & - & - & - & - & - & - & & & \\
\hline
\end{tabular}

$\mathrm{M}=$ milho; $\mathbf{S}=$ soja; $\mathrm{A}=$ algodz̃o; $\mathrm{C}=$ plantio contínuo. 
Quadro 6. Produtividade de milho em rotação com algodão e soja, resultados do teste $F$ na análise da variância e do teste de combinação de probabilidades $\left(\chi^{2}\right)$, em Ribeirão Preto (SP)

\begin{tabular}{|c|c|c|c|c|c|c|c|}
\hline \multirow{2}{*}{ Rotação } & \multicolumn{5}{|c|}{ Anos agrícolas } & \multirow{2}{*}{ Média } & \multirow{2}{*}{$\begin{array}{r}\text { Produção } \\
\text { relativa }\end{array}$} \\
\hline & $84 / 85$ & $85 / 86$ & $86 / 87$ & $87 / 88$ & $88 / 89$ & & \\
\hline & \multicolumn{5}{|c|}{ kg/ha } & 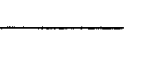 & $\%$ \\
\hline S-A-M & 6.080 & 6.829 & 5.831 & 5.850 & 5.764 & 6.071 & 123 \\
\hline S-M-M & 6.246 & 5.462 & 5.868 & 5.883 & 3.790 & 5.450 & 111 \\
\hline M-S-M & 5.830 & 6.508 & 4.540 & 6.555 & 4.648 & 5.616 & 114 \\
\hline S-S-M & 6.101 & 6.363 & 6.112 & 5.673 & 4.056 & 5.661 & 115 \\
\hline Milho-C & 5.533 & 4.843 & 4.081 & 5.202 & 4.925 & 4.918 & 100 \\
\hline Média & 5.958 & 6.001 & 5.286 & 5.833 & 4.637 & 5.543 & \\
\hline CV\% & 13,6 & 18,3 & 9,7 & 8,0 & 25,8 & 13,7 & \\
\hline Contrastes & & & & & Anál & conjunta & \\
\hline C vs. rotação & ns & $\mathbf{n s}$ & $*$ & ns & ns & $*$ & \\
\hline S-A-M vs. S-S-M & ns & ns & ns & ns & ns & ns & \\
\hline S-M-M vs. M-S-M & ns & ns & ns & ns & ns & ns & \\
\hline Anos X rotação & - & - & - & - & - & - & \\
\hline S-A-M vs. C & & & & & $\chi^{2}$ & $28,30^{*}$ & \\
\hline S-M-M vs. C & & & & & $x^{2}$ & $23,69^{*}$ & \\
\hline M-S-M vs. C & & & & & $x^{2}$ & $22,31^{*}$ & \\
\hline S-S-M vs. C & & & & & $\chi^{2}$ & $31,08^{*}$ & \\
\hline
\end{tabular}

$\mathrm{M}=$ milho; $\mathbf{S}=$ soja; $\mathrm{A}=$ algodão; $\mathrm{C}=$ plantio contínuo.

Quadro 7. Produtividade de algodão em caroço em rotação com milho e soja, resultados do teste $\mathrm{F}$ na análise da variância e do teste de combinação de probabilidades $\left(\chi^{2}\right)$, em Ribeirão Preto (SP)

\begin{tabular}{|c|c|c|c|c|c|c|c|}
\hline \multirow{2}{*}{ Rotação } & \multicolumn{5}{|c|}{ Anos agricolas } & \multirow{2}{*}{ Média } & \multirow{2}{*}{$\begin{array}{l}\text { Produção } \\
\text { relativa }\end{array}$} \\
\hline & $84 / 85$ & $85 / 86$ & $86 / 87$ & $87 / 88$ & $88 / 89$ & & \\
\hline & \multicolumn{6}{|c|}{$-\mathbf{k g} / \mathbf{h a}$} & $\%$ \\
\hline M-S-A & 2.030 & 2.550 & 3.197 & 2.921 & 2.722 & 2.680 & 106 \\
\hline S-A-A & 1.600 & 2.120 & 3.654 & 2.523 & 3.092 & 2.598 & 102 \\
\hline A-S-A & 1.676 & 2.141 & 3.494 & 2.669 & 2.704 & 2.537 & 100 \\
\hline S-S-A & 1.767 & 2.743 & 3.382 & 2.546 & 3.141 & 2.715 & 107 \\
\hline Algodão-C & 1.629 & 1.948 & 3.264 & 2.988 & 2.891 & 2.544 & 100 \\
\hline Média & 1.741 & 2.300 & 3.398 & 2.729 & 2.910 & 2.616 & \\
\hline CV\% & 17,4 & 16,3 & 18,4 & 14,0 & 13,1 & 17,2 & \\
\hline
\end{tabular}

Continua 
Quadro 7. Conclusão

Anos agrícolas

Rotação

Média

Produção

$84 / 85$

$86 / 87$

$87 / 88$ relativa

\section{Contrastes}

C vs. rotação

M-S-A vs. S-S-A

S-A-A vs. A-S-A

Anos $X$ rotação

$85 / 86$

$\mathbf{8 7 8 8}$

$+2$

$\mathbf{M}=$ milho; $\mathbf{S}=$ soja; $\mathbf{A}=$ algodăo; $\mathbf{C}=$ plantio continuo.

Quadro 8. Produtividade de soja em rotação com algodão e milho, resultados do teste $\mathrm{F}$ na análise da variância e do teste de combinação de probabilidades $\left(\chi^{2}\right)$, em Ribeirão Preto (SP)
Análise conjunta

$\begin{array}{cc}\text { ns } & \text { ns } \\ \text { ns } & \text { ns } \\ \text { ns } & \text { ns } \\ - & -\end{array}$

s

s

$-$ ns ns

ns

ns

ns

ns

\begin{tabular}{|c|c|c|c|c|c|c|c|}
\hline \multirow{2}{*}{ Rotação } & \multicolumn{4}{|c|}{ Anos agrícolas } & \multirow[b]{2}{*}{$88 / 89$} & \multirow{2}{*}{ Média } & \multirow{2}{*}{$\begin{array}{c}\text { Produção } \\
\text { relativa }\end{array}$} \\
\hline & $84 / 85$ & $85 / 86$ & $86 / 87$ & $87 / 88$ & & & \\
\hline & \multicolumn{5}{|c|}{ - kg/ha } & & $\%$ \\
\hline A-M-S & 2.602 & 1.616 & 2.471 & 2.720 & 2.495 & 2.391 & 107 \\
\hline A-A-S & 2.701 & 1.962 & 2.682 & 2.776 & 2.363 & 2.497 & 112 \\
\hline M-M-S & 2.360 & 1.961 & 2.736 & 2.952 & 2.441 & 2.490 & 112 \\
\hline M-S-S & 2.656 & 1.743 & 2.458 & 2.720 & 1.912 & 2.298 & 103 \\
\hline S-M-S & 2.373 & 1.863 & 2.542 & 2.986 & 2.502 & 2.453 & 110 \\
\hline A-S-S & 2.575 & 1.691 & 2.396 & 2.911 & 2.000 & 2.314 & 103 \\
\hline S-A-S & 3.434 & 1.691 & 2.549 & 3.016 & 2.341 & 2.386 & 107 \\
\hline Soja-C & 2.402 & 1.490 & 2.391 & 2.985 & 1.881 & 2.330 & 100 \\
\hline Média & 2.500 & 1.759 & 2.528 & 2.883 & 2.242 & 2.382 & \\
\hline CV\% & 11,5 & 18,9 & 9,1 & 6,5 & 12,7 & 11,9 & \\
\hline
\end{tabular}

\section{Contrastes}

C vs. rotação

A-M-S vs. A-A-S + M-M-S

M-S-S vs. S-M-S

A-S-S vs. S-A-S

A-A-S vs. M-M-S

Anos X rotação 
Analisando as produções relativas, as rotações algodão-algodão-soja e milho-milho-soja apresentaram as maiores produções, seguidos por soja-milho-soja, soja-algodão-soja e algodão-milho-soja. Os de menores rendimentos foram os sistemas correspondentes a algodão-soja-soja e milho-soja-soja. A soja e o algodão não responderam às rotaçōes trienais.

\section{CONCLUSÕES}

1. Nas duas localidades, houve respostas significativas do milho na rotação com soja e algodão.

2. O algodão e a soja não responderam aos sistemas de rotação estudados.

\section{AGRADECIMENTOS}

Ao Dr. Francisco Lombardi Neto, da Seção de Conservação do Solo, pelo auxílio na escolha dos tratamentos.

\section{REFERÊNCIAS BIBLIOGRÁFICAS}

BERTONI, J.; PASTANA, F.I.; LOMBARDI NETO, F. \& BENATTI JUNIOR, R. Conclusóes gerais das pesquisas sobre conservação do solo, no Instituto Agronómico. Campinas, Instituto Agronômico, 1972. 56p. (Circular, 20)

CONAGIN, A.; IGUE, T. \& NAGAI, V. Teste de combinação de probabilidades em grupos de experimentos. Campinas, Instituto Agronômico, 1993. 10p. (Boletim científico, 32)
GALLO, P.B.; LAVORENTI, A.; SAWAZAKI, E.; HIROCE, R. \& MASCARENHAS, H.A.A. Efeito de cultivos anteriores de soja na produção e no teor de nitrogênio das folhas e dos grãos de milho. Revista Brasileira de Ciência do Solo, Campinas, 5(1):64-67, 1981.

MASCARENHAS, H.A.A.; HIROCE, R.; BRAGA, N.R.; MIRANDA, M.A.C. de; POMMER, C.V. \& SAWAZAKI, E. Efeito do nitrogénio residual de soja na produção do milho. Campinas, Instituto Agronômico, 1978. 16p. (Boletim técnico, 58)

NEME, N.A. Ensaios comparativos de adubos verdes. In: INSTITUTO AGRONÔMICO (Campinas). Relatório da Seção de Leguminosas: 1959. Campinas, 1960. 10p. (Datilografado)

OLIVEIRA, O.F. de; FELICIO, J.C.; MASCARENHAS, H.A.A. \& HIROCE, R. Efeito do nitrogênio residual da soja na produção de trigo. Bragantia, Campinas, 38:LVII-LIX, 1979. (Nota, 13)

PEREIRA, J.C.V.N.A.; MASCARENHAS, H.A.A.; HIROCE, R. \& CAMARGO, O.B.A. Efeitos do nitrogênio e da rotação com soja na produção de arroz-de-sequeiro. Bragantia, Campinas, 38:LIII-LVI, 1979. (Nota, 12)

PEREIRA, J.C.V.N.A.; MASCARENHAS, H.A.A.; MARTINS, A.L.M.; BRAGA, N.R.; SAWAZAKI, E. \& GALLO, P.B. Efeito da adubação nitrogenada em cobertura no cultivo contínuo do milho e do algodão e em rotação com soja. Revista de Agricultura, Piracicaba, 63(1):95-108, 1988.

STICKLER, F.C.; SHRADER, W.D. \& JOHNSON, I.J. Comparative value of legume and fertilizer nitrogen for corn production. Agronomy Journal, Madison, 51(3):157-160, 1959.

SUTHERLAND, W.N.; SHRADER, W.D. \& PESEK, J.T. Efficiency of legume residue nitrogen and inorganic nitrogen in corn production. Agronomy Journal, Madison, 53(5):339-342, 1961. 